\title{
EFFÍCIENT PAPR REDUCTION APPROACH FOR SELECTIVE MAPPING İN A STBC MIMO-OFDM SYSTEM
}

\author{
VijayaLakshmi $\mathrm{M}^{1}$ and K Rama Linga Reddy ${ }^{2}$ \\ ${ }^{1}$ ETM Dept, GNITS, Hyderabad, India \\ ${ }^{2}$ HOD, ETM Dept, GNITS, Hyderabad, India
}

\begin{abstract}
The demand for wireless communication increases every day. MIMO system increases the channel capacity without increasing the bandwidth. OFDM eliminates inter-symbol interference very effectively. By combining both these technologies, the resultant data rates are increased and also robustness to multi path fading effects. But the main drawback of MIMO-OFDM system is its high PAPR. Selective mapping(SLM) is one of the popular distortion less technique for reducing high PAPR but the computational complexity for searching optimum phase factors is high. Many evolutionary algorithms like particle swarm optimisation, artificial bee colony optimization algorithm, cuckoo search algorithm are proposed for searching optimum phase factors. In this paper two new optimization algorithms social spider optimization(SSO), adaptive artificial bee colony algorithm are proposed for SLM and Simulation results shows that compared to adaptive artifctial bee colony algorithm, social spider optimization algorithm(SSO) gives best phase factors for lower PAPR with fewer number of iterations and also reduces the computational complexity of SLM technique very efficiently.
\end{abstract}

\section{KEYWORDS}

Multiple input multiple output(MIMO), orthogonal frequency division multiplexing (OFDM),peak to average power ratio(PAPR). Space time block codes $(S T B C)$.

\section{INTRODUCTION}

In wireless communication, a remarkable development is a combination of multiple-input multiple-output (MIMO) with orthogonal frequency division multiplexing (OFDM).MIMO OFDM has the potential to achieve high system capacity and more diversity at transmitter and receiver for reliable communication of wireless system. STBC is used to improve the reliability of the wireless communication system and the performance of STBC decoder is similar to the maximum ratio combining without the need of channel state information at the receiver. OFDM has been adopted as a standard technique for WLAN, WiMAX, LTE. But the main disadvantage of MIMO-OFDM is high PAPR. The OFDM signal consists of many independently modulated subcarriers and is spaced very closely, which may lead to a large PAPR for some OFDM symbols. Due to high PAPR, power amplifier at the transmitter enters into non linear region and because of this it generates nonlinear distortion. High PAPR results in increased inter symbol interference, high out of band radiation. To avoid high PAPR effects linear amplifiers with large dynamic range is required and these are very expensive.

Several schemes were proposed to diminish the large PAPR such as distortion less techniques and distortion techniques, coding techniques. Under distortion techniques Clipping is simple technique for reducing high PAPR and it also generates out of band emission and in-band distortion. Tone reservation is also distortion less technique but it requires some additional

DOI : 10.5121/ijcnc.2018.10304 
bandwidth for pilots. Out of all PAPR reduction techniques, distortion less techniques are very popular and in this partial transmit sequence and selective mapping are efficient techniques. But the complexity of searching optimum phase sequences is high in both PTS,SLM PAPR reduction techniques. Many optimization algorithms are proposed for reducing the complexity of SLM and PTS techniques. Differential Evolution[1] is simple and powerful search technique for optimization problems and it is applied to the PTS for reducing the complexity of searching best phase sequence. Artificial bee colony algorithm[2], [3] is an intelligent swarm-based algorithm and it is proposed for reducing the complexity of PTS in MIMO-OFDM system with solid state power amplifier and travelling wave tube power amplifier. cuckoo[4], modified cuckoo[5], ant colony algorithm[6]s are also applied for reducing the complexity of partial transmit sequence. Efficient hybrid algorithms like cuckoo with $\mathrm{DE}[7], \mathrm{ABC}$ with $\mathrm{DE}[8]$ are proposed for optimizing the large-scale problems.

\section{LITERATURE REVIEW}

Maha Cherif Dakhli et al.[9] have declared a new compensator for reducing non-linear distortion generated by power amplifier. This approach is based on neural networks with MMSE.In this method compensation is applied at the transmitter before the power amplifier and after OFDM demodulation at the receiver. Due to nonlinear distortion system capacity is reduced but with new compensation method it becomes close to that off linear case.

Pawan Sharma et al.[10] have declared a method for improving the performance of SLM by adding turbo codes..Traditional SLM method reduces PAPR with high complexity and it also requires side information for recovering the optimum phase sequences. But in this SLM with turbo codes, side information is not required and it also improves the BER performance along with PAPR reduction.

Tarik Hadj Ali et al.[11] have declared a method for reducing the computational complexity of SLM for searching the optimum phase sequences which are used to reduce the PAPR. He proposed a new genetic algorithm for searching the optimum phase sequences with few numbers of iterations. This new method SLM with GA impoves the performance of SLM with lower complexity.

Necmi Tasspinar et al [12] have declared a method for reducing the complexity of SLM for obtaining best phase sequences to reduce the high PAPR of OFDM signal. In this approach improved $\mathrm{ABC}$ algorithm is applied for searching best phase sequences with lower complexity. In this proposed method PAPR performance of OFDM signal is reduced with the lowest complexity. Erik Cuevas1 et al [13] have declared a new swarm optimization algorithm called social spider optimization algorithm for solving optimization tasks.It is based on the simulation of the cooperative behavior of social spiders. The performance of this optimization algorithm high for searching global optimum with several bench mark functions.

In this paper social spider optimisation algorithm,adaptive artificial bee colony algorithm is applied to reduce the complexity for searching the optimum phase sequences in SLM method, which in turn used to reduce the peak to average power reduction ratio in MIMO-OFDM . 


\subsection{MIMO-OFDM}

Let $\mathrm{X}=\left[\mathrm{X}_{0}, \mathrm{X}_{1}, \ldots \ldots . \mathrm{X}_{\mathrm{N}-1}\right]$ denotes the input data symbol vector of size $\mathrm{N}$. Each $\mathrm{X}_{\mathrm{k}}$ represents the modulated PSK symbols or QAM symbols. The data symbol vector $\mathrm{X}$ is applied to the input of STBC MIMO system. The output of STBC MIMO system are represented by

$$
\begin{gathered}
X_{M I M O, 1}=\left[X_{0},-X_{1}^{*}, \ldots \ldots . X_{N-2,}-X_{N-1}^{*}\right] \\
X_{M I M O, 2}=\left[X_{1}, X_{O}^{*}, \ldots \ldots \ldots X_{N-1}, X_{N-2}^{*}\right]
\end{gathered}
$$

Where $(.)^{*}$ represents the complex conjugate operation.

The output of MIMO system is applied to the input of OFDM system and IFFT is applied to the modified symbol sequence with $\mathrm{N}$ no. of subcarriers. The output of the OFDM system from the first MIMO path is

$x_{O F D M 1}(n)=\frac{1}{\sqrt{N}} \sum_{k=1}^{N-1} X_{M I M O, 1}(k) \cdot e^{j 2 \pi k n / N}, \quad n=0, \ldots \ldots . N-1$

Similarly $\mathrm{X}_{\mathrm{MIMO}, 2}$ is also applied to get the other OFDM signal.

The PAPR or peak factor of OFDM signal is defined as

$$
P A P R \text { of } x_{O F D M}=\frac{\max |x(n)|^{2}}{E[x(n)]^{2}}
$$

Where E[.] represents expectation operation. The parameter complementary cumulative distribution function (CCDF) is used to measure the PAPR of OFDM signal and it represents probability of PAPR exceeds the threshold value $p_{0}$ which is given by

$$
\operatorname{prob}\left(P A P R>p_{0}\right)=1-\left(1-e^{-p_{0}}\right)^{\alpha N}
$$

Where $\alpha=1$ for $\mathrm{N} \geq 512$ and $\alpha=0.2 .8$ for $\mathrm{N} \leq 256$.

\subsection{SelectiveMapping:}

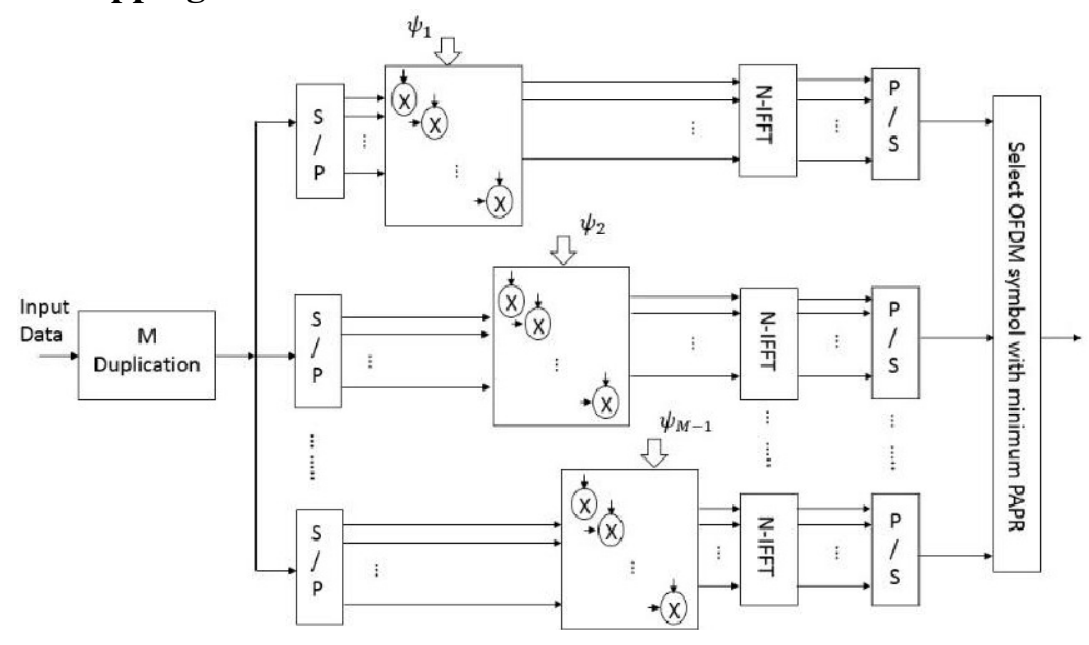


Figure.1 Block diagram of selective mapping

In this SLM approach, the data symbol sequence $X=X_{0}, X_{1} \ldots \ldots . X_{N-1}$ are multiplied with phase sequence $U=U_{0}, U_{1}, \ldots \ldots \ldots U_{N-1}$. Each phase sequence element $U_{K}$ can take any value from the set of predefined set of phase factor values.

$$
\begin{array}{cc}
U_{K}=e^{j * \emptyset} \text { where } & \emptyset \in\{0,2 \pi\} \\
& U_{K}= \pm 1 \text { if } w=2
\end{array}
$$

$U_{K}= \pm 1, \pm j$ if $w=4$

Where $w$ indicates the no .of phase factors used in the SLM system.

The output of SLM after multiplying OFDM data symbol sequence with phase sequence is

$V_{p}=\left(X_{0} U_{0}, X_{1} U_{1} \ldots \ldots \ldots, X_{N-1} U_{N-1}\right)$ where $p=1$ to $\mathrm{w}^{N-1}$.

Since the total no.of phase sequences are $w^{N-1}$ and $U_{o}$ is always unity.SLM selects the lowest PAPR signal out of all $w^{N-1}$ signals and it is very complex process even for smaller values of $\mathrm{N}$ and $w$.To reduce the complexity of SLM for searching of optimum phase sequences Adaptive $\mathrm{ABC}$, social spider optimization algorithm are applied to the SLM and also compared in this paper.

\section{Proposed Methods}

\subsection{Adaptive ABC Algorithm:}

It is a hybrid algorithm of $\mathrm{ABC}$ and cuckoo algorithm. In $\mathrm{ABC}$ algorithm food positions represents the phase sequences and these are initialized randomly. Nectar amount of food source represents the fitness of the solution and High fitness nest gives the lowest PAPR solution. In this algorithm, honey bees are categorized into employed bees, onlooker bees, and scout bees. Initially, employed bees are represented by the phase sequences and these are assigned randomly. Each phase sequence consists of $\mathrm{N}$ no. of elements. The no. of employed bees represents the population size p. Each employed bee finds the nearest new food source and it replaces if the new source holds high fitness otherwise it holds the previous food position. For each employed bee new food source is expressed as

$$
b_{i}^{\prime}=b_{i}+\emptyset_{i}\left(b_{i}-b_{k}\right) \text { where } \mathrm{i}=1 \text { to } \mathrm{P}
$$

$\mathrm{k}$ is a random number which is not equal to $\mathrm{i}$ and is within the same range of $\mathrm{i}, \emptyset_{i}$ is also a random number in the range of $[-1,1]$.Each employed bee share the fitness i.e PAPR of the phase sequene with the onlooker bee.

$$
\begin{aligned}
\operatorname{fitness}\left(b_{i}\right)= & \frac{1}{1+f\left(b_{i}\right)} \quad \text { for } f\left(b_{i}\right) \geq 0 \\
& =1+\operatorname{abs}\left(f\left(b_{i}\right) \quad \text { for } f\left(b_{i}\right)<0\right.
\end{aligned}
$$

Where $f\left(b_{i}\right)$ represents the PAPR value. 
The onlooker bee moves to a food source with the probability

$$
\operatorname{prob}_{i}=\frac{\operatorname{fitness}\left(b_{i}\right)}{\sum_{i=1}^{N} \operatorname{fitness}\left(b_{i}\right)}
$$

Onlooker bee searches for the new food source

within the neighbourhood of the previous food source and it memorizes the food source which is having the best fitness i.e lowest PAPR. Employee bees, onlooker bees repeats the same procedure till the no.of iterations reaches 'limit'

value. If the best source is not discovered within the 'limit' no. of iterations, then employee bee converts into scout bees. Now scout bees searches the best food source with the help of Levy flight parameter(from cuckoo search) and is given by .

$$
b_{i}^{\prime}=b_{i}+\delta \oplus \operatorname{levy}(\beta)
$$

Where $\delta$ is a constant and it is usually equal to 1 and $\oplus$ represents the entry wise multiplications. Levy flight is an efficient search solution and it gives long step size in long run.

$$
\text { levy u }=t^{-\lambda}, \quad(1<\lambda \leq 3)
$$

\subsection{Social spider optimization algorithm:}

This optimisation algorithm starts by defining total no. of population(p) i,e total no. of spiders which represents the random selection of small set of phase sequences from the large set of $w^{N-1}$ phase sequences.

The total no. of population $p$ is divided into male spiders $p_{m}$ and female spiders $p_{f}$.

The no. of female spiders $\left(p_{f}\right)$ are $65 \%-90 \%$ of entire population and it is calculated with the help of following equation

$$
p_{f}=\text { floor }[(0.9-\text { rand } .0 .25) . s]
$$

The no. of male spiders $p_{m}$ are calculated by using the formula

$$
p_{m}=p-p_{f}
$$

Initially each spider position is selected randomly and it is uniformly distributed between the fixed lower limit(LL) and upper limit(UL).

The dimensions of LL and UL are similar to the data size.

$$
\begin{gathered}
f_{i, j}^{k}=L L_{j}+\operatorname{rand}(0,1) \cdot\left(U L_{j}-L L_{j}\right) \\
\text { where } \quad i=1,2, \ldots \ldots . . p_{f} j=1,2, \ldots . N \\
m_{l, j}^{k}=L L_{j}+\operatorname{rand}(0,1) .\left(U L_{j}-L L_{j}\right) \\
\text { where } \quad l=1,2, \ldots \ldots . p_{m} j=1,2, \ldots . N
\end{gathered}
$$

The complete set of spider population S=FUM where F\&M represents the sets of female population and male population. 
International Journal of Computer Networks \& Communications (IJCNC) Vol.10, No.3, May 2018

$\mathrm{F}=\left[f_{1}, f_{2}, \ldots . f_{p_{f}}\right], M=\left[m_{1}, m_{2} \ldots \ldots m_{p_{m}}\right], S=\left[s_{1}=f_{1}, s_{2}=f_{2}, \ldots, s_{p_{f}}=f_{p_{f}}, s_{p_{f+1}}=\right.$ $\left.m_{1}, \ldots . s_{p}=m_{p_{m}}\right]$.

For each spider $\left(\mathrm{s}_{\mathrm{i}}\right)$ weight $\left(d_{i}\right)$ is assigned based on the fitness value. Here fitness represents the PAPR value.

$$
d_{i}=\frac{\text { fitness of } s_{i}-\text { worst fitness }}{\text { best fitness }- \text { worst fitness }}
$$

Worst fitness represents the highest PAPR from and best fitness represents the lowest PAPR from the set of all $\mathrm{S}$ phase sequences.

The communal web is used to exchange information between spiders through vibrations. The PAPR information is encoded in the form of small vibrations that are critical for the collective coordination of all members in the population. Vibrations are three types and these are depend on the individual weights and Euclidian distance between the spiders.

$v i b c_{i}$ represents the information collected by spider $\left(\mathrm{s}_{\mathrm{i}}\right)$ from the spider $\mathrm{s}_{\mathrm{c}}$ which is nearest and also holds higher weight compared to the $\mathrm{s}_{\mathrm{i}}$.

$$
v i b c_{i}=d_{c} \cdot e^{-d_{i, c}^{2}}
$$

$v i b b_{i}$ represents the information collected by spider $\left(\mathrm{s}_{\mathrm{i}}\right)$ from the spider $\mathrm{s}_{\mathrm{b}}$ which is having best weight from the set of $\mathrm{S}$.

$$
v i b b_{i}=d_{b} \cdot e^{-d_{i, b}^{2}}
$$

$v i b f_{i}$ represents the information collected by spider $\left(\mathrm{s}_{\mathrm{i}}\right)$ from the spider $\mathrm{s}_{\mathrm{f}}$ which is nearest female spider .

$$
v i b f_{i}=d_{f} \cdot e^{-d_{i, f}^{2}}
$$

Now new female spiders are generated depending on female co-operator and some random number ' $r$ ' which is varying from $[0,1]$.

$f_{i}^{k+1}=f_{i}^{k}+\alpha \cdot v i b c_{i} \cdot\left(s_{c}-f_{i}^{k}\right)+\beta \cdot v i b b_{i} \cdot\left(s_{b}-f_{i}^{k}\right)+\delta .(r a n d-0.5) \quad$ (19) if $\mathrm{r}$ is less than threshold.

$f_{i}^{k+1}=f_{i}^{k}-\alpha \cdot v i b c_{i} .\left(s_{c}-f_{i}^{k}\right)-\beta \cdot v i b b_{i} \cdot\left(s_{b}-f_{i}^{k}\right)+\delta .(r a n d-0.5) \quad(20)$ if $\mathrm{r}$ is greater than threshold.

$\alpha, \beta, \delta$ and rand are random numbers between $[0,1]$, where $\mathrm{k}$ represents the iteration number. Similarly new male spiders are generated according to the male co-operator and some random number ' $r$ ' which is varying from $[0,1]$.

$$
\begin{aligned}
& m_{i}^{k+1}=m_{i}^{k}+\alpha . v i b f_{i} \cdot\left(s_{f}-m_{i}^{k}\right)+\delta .(\text { rand }-0.5) \text { if } d_{p_{f}+i}>d_{p_{f+m}} \\
& m_{i}^{k+1}=m_{i}^{k}+\alpha \cdot \frac{\sum_{h=1}^{p_{m}} d_{p_{f+h}} m_{h}^{k}}{\sum_{h=1}^{p_{m}} d_{p_{f}}+h}-m_{i}^{k} \quad \text { if } \quad d_{p_{f}+i} \leq d_{p_{f+m}}
\end{aligned}
$$

Now dominant male of having lowest PAPR mates with nearest female spider within in the mating range and produces new spiders. The radius of mating is computed according to following formula

$$
r=\frac{\sum_{j=1}^{N} U L_{j}-L L_{j}}{2 N} .
$$


International Journal of Computer Networks \& Communications (IJCNC) Vol.10, No.3, May 2018

The PAPR of new spider is calculated and it is compared with worst PAPR of the spider colony members .If the new spider PAPR is less than the worst then it is replaced otherwise it is discarded. This process is repeated for specified number of iterations or until target lowest PAPR is reached.

\section{Simulation RESUlts}

The analysis of the Proposed SLM with SSO and adaptive ABC methods have been carried out using MATLAB 8.6.0.267246 (R2015b).The simulation parameters considered for this analysis are summarized in Table 1.

Table 1. Simulation Parameters

\begin{tabular}{|l|l|}
\hline Simulation Parameters & Type/ Values \\
\hline Number of random OFDM blocks & 10000 \\
\hline Number of subcarriers & 512,1024 \\
\hline Oversampling factor(L) & 4,8 \\
\hline Modulation scheme & QPSK \\
\hline Optimal Phase weighting factor (b) & $1,-1, \mathrm{j},-\mathrm{j}$ \\
\hline Population size & 10 \\
\hline
\end{tabular}

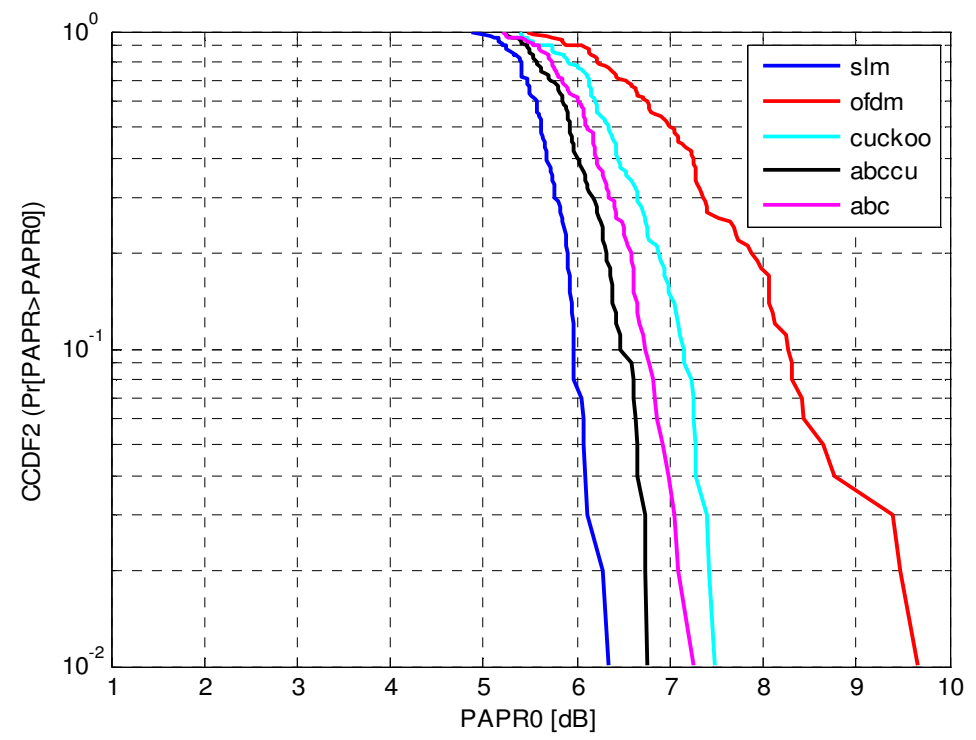

Figure.2 PAPR Comparison of OFDM,Cuckoo,ABC,Adaptive ABC,SLM

Figure. 2 illustrates the performance of adaptive $A B C$ for lessening of PAPR. When $C C D F=0$, the PAPR of OFDM is $9.8 \mathrm{~dB}$, for ABC PAPR is $7.5 \mathrm{~dB}$, for cuckoo search PAPR is $7.2 \mathrm{~dB}$,Adaptive $\mathrm{ABC}(\mathrm{ABC}$ with Cuckoo) PAPR is $6.8 \mathrm{~dB}$.For the conventional PAPR is $6.4 \mathrm{~dB}$ but it requires $(64)^{\wedge} 4$ iterations and the other systems requires only 20 iterations with population size 10. 
International Journal of Computer Networks \& Communications (IJCNC) Vol.10, No.3, May 2018

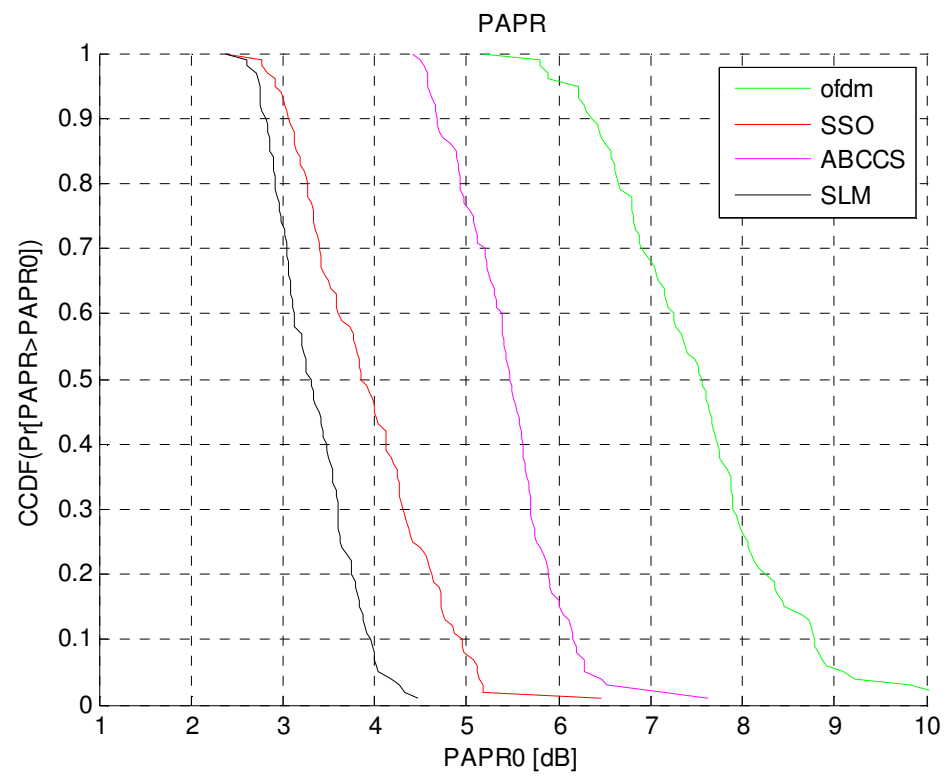

Figure.3 PAPR Comparison for SSO,ABCCS,SLM,OFDM

The Figure. 3 illustrates the performance of SSO for lessening the PAPR. When CCDF=0, the PAPR of the MIMO-OFDM is $9.3 \mathrm{~dB}$, and for SLM is $4.2 \mathrm{~dB}$, and in conclusion the PAPR of the SSO- SLM is $5.1 \mathrm{~dB}$ and ABCCS-SLM is $7.4 \mathrm{~dB}$.But conventional SLM requires (64)^4 iterations and the SSO requires only 20 iterations with population size 10 . So the computational complexity of SSO-SLM is lower than the computational complexity of conventional SLM.

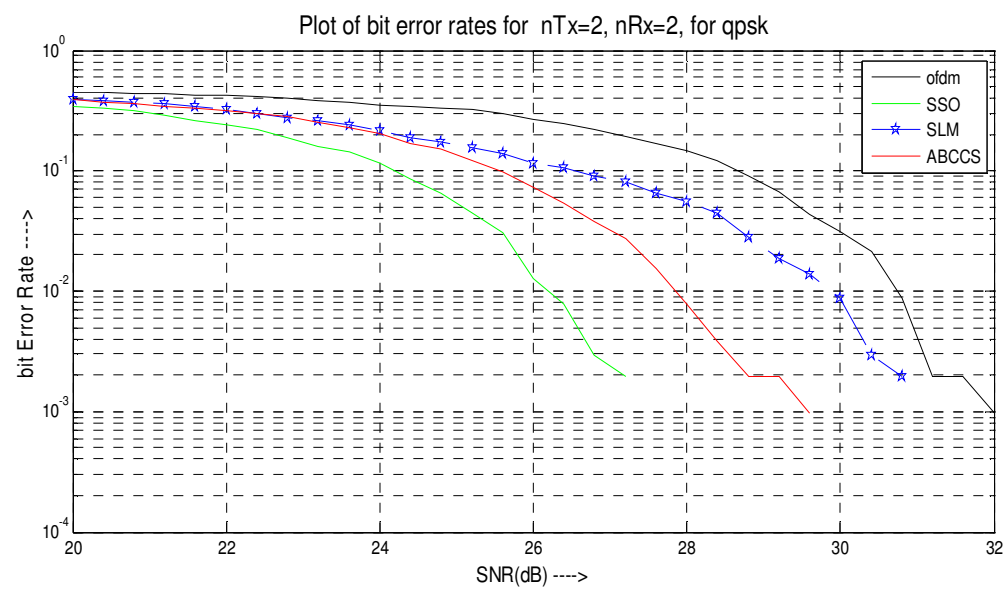

Figure.4 BER Comparison

Figure.4 illustrate the BER performance of MIMO-OFDM, conventional SLM,SLM combined SSO and ABSCS with nonlinear power amplifier and in conclusion BER of SLM with SSO is better than the BER of SLM with ABCCS and conventional SLM and MIMO-OFDM. 
International Journal of Computer Networks \& Communications (IJCNC) Vol.10, No.3, May 2018

\section{CONCLUSIONS}

Selective mapping method is used to diminish the PAPR of MIMO-OFDM system. But SLM requires more complex searches for finding the optimum solutions. Adaptive ABC, social spider algorithms are used to reduce the no.of complex searches required in conventional SLM. The performance of SSO is superior compared to the adaptive ABC algorithm and it can be further improved by taking more no.of phase factors or by combining SSO with some other algorithm.

\section{REFERENCES:}

[1] Yung-cheng yao.,Ho-lung hung."PAPR reduction in OFDM system differential evolution based partial transmit sequence scheme".proceedings of the international conference on machine learning and cybermetics, Guilin,july 2011,10-13

[2] Karaboga,D.,Basturk,B.“A powerful and efficient algorithm for numerical function optimization: artificial bee colony (ABC) algorithm”, Journal of Global Optimization, Vol. 39, pp. 459-471, 2007.

[3] Necmi,TAS.,PINAR1,Dervi.,KARABO,S.,GA2."Mahmut,YILDIRIM3.,Bahriye ，Akay2.:PAPR reduction using artificial bee colony algorithm in OFDM systems", Turk j elec eng\&comp sci,vol.19,No.1,2011

[4] Manjith,R.,suganthi,M. “ peak to average power ratio reduction using modified cuckoo search algorithm in MIMO-OFDM systems". Australian journal of basic and applied sciences,7(13),November,2013,pages 32-43.

[5] Mangal,singh.,sarat kumar,patra. "Partial transmit sequence based cuckoo search optimization for peak to average power ratio reduction in orthogonal frequency division multiplexing",jcies 2016.1134,DOI 10.1166.

[6] Devinder,Kumar.,Preethi,singh. "complexity reduction in PTS based OFDM system: A Survey”.International journal of computer applications(0975-8887),volume 69,no 13may 2013

[7] Asgarali,Bouyer.,Habib,Ghafarzadeh.,Omid,Tarkhaneh. "An efficient hybrid algorithm using cuckoo search and differential evolution for data clustering", Indian Journal of Science and Technology, Vol 8(24), DOI: 10.17485/ijst/2015/v8i24/60146, September 2015

[8] Nadezda,Stanarevic."Hybridizing Artifitial bee colony algorithm with differential evolution for large scale optimization problems". International journal of mathematics and computers in simulation, Issue 1, Volume 6, 2012

[9] Maha,Cherif.,Dakhli.,Rafik,Zayani.,Ridha,Bouallegue."BER Analysis and Compensation for the Effects of Polynomial HPA Non-linearity in MIMO OFDM Systems Over Fading Channel”. Wireless Personal Communications, vol. 81, no. 1, pp. 133-149, 2014.

[10] Pawan,sharma.,Veema.Varma,“" papr reduction of ofdm signals using selective mapping with turbo codes”. International Journal of Wireless \& Mobile Networks (IJWMN) Vol. 3, No. 4, August 2011

[11] Tarik,Hadj Ali.,Hamza,A.,LISIC. "A new SLM technique based on Genetic Algorithms for PAPR reduction in OFDM systems". International journal of communications ,Volume 10, 2016ISSN

[12] Necmi,Ta.,spınar,Mahmut Yıldırım. “A Novel Parallel Artificial Bee Colony Algorithm and Its PAPR ReductionPerformance Using SLM Scheme in OFDM and MIMO-OFDM Systems",IEEE communications letters, vol. 19, no. 10, october 2015

[13] Cuevas,E. "A swarm optimization algorithm inspired in the behavior of the social-spiderbehavior Expert Systems with Applications”, 40 (16), (2013), pp. 6374-6384 